\title{
Minireview
}

\section{DCE-MRI biomarkers in the clinical evaluation of antiangiogenic and vascular disrupting agents}

\author{
JPB O'Connor*, I,2, A Jackson', GJM Parker' and GC Jayson ${ }^{2}$ \\ IImaging Science and Biomedical Engineering, University of Manchester, Oxford Road, Manchester MI 3 9PT, UK; ${ }^{2}$ Cancer Research UK Department of \\ Medical Oncology, Christie Hospital, Wilmslow Road, Manchester M20 4BX, UK
}

Dynamic contrast-enhanced magnetic resonance imaging (DCE-MRI) is now frequently used in early clinical trial assessment of antiangiogenic and vascular disrupting compounds. Evidence of drug efficacy and dose-dependent response has been demonstrated with some angiogenesis inhibitors. This review highlights the critical issues that influence $T_{1}$-weighted DCE-MRI data acquisition and analysis, identifies important areas for future development and reviews the clinical trial findings to date.

British Journal of Cancer (2007) 96, I89- 195. doi:I0.1038/sj.bjc.66035I5 www.bjcancer.com

Published online 9 January 2007

(c) 2007 Cancer Research UK

Keywords: angiogenesis inhibitors; biomarkers; clinical trials; dynamic contrast-enhanced magnetic resonance imaging; image analysis

\section{ANGIOGENESIS: A TARGET FOR ANTICANCER THERAPY}

In order to survive and grow beyond a few hundred micrometers, tumours require adequate oxygen and nutrient delivery, as well as removal of waste products. Angiogenesis, the process by which tumours develop a circulatory blood supply, results in the development of vascular networks that are both structurally and functionally abnormal. Compounds that disrupt new vessel formation (antiangiogenic) or destroy existing vessels (vascular disrupting) offer potential targets for novel anticancer therapy (Baluk et al, 2005). This strategy has been validated by recent studies of the anti-vascular endothelial growth factor (VEGF) antibody bevacizumab, which have shown improvement in clinical outcome in phase III randomised-controlled trials in colorectal (Hurwitz et al, 2004) and other common human cancers.

Angiogenesis inhibitors in clinical development pose challenges for phase I/II trial design. Because they reduce tumour growth or prevent metastases through primarily cytostatic modes of action such as, selectively inhibiting membrane receptors, cell cycle regulators or other signalling pathways, conventional end points based on reduction in tumour size may be inadequate for evaluating clinical response. Alternative imaging biomarkers of angiogenesis are being sought, which can serve as early indicators of drug activity in clinical trials and may facilitate early pharmacodynamic assessments by speeding up the go/no-go decision-making process (Jayson and Waterton, 2005).

Dynamic contrast-enhanced magnetic resonance imaging measurements have been incorporated as biomarkers of drug efficacy in clinical trials of angiogenesis inhibitors. The technique is

\footnotetext{
*Correspondence: Dr JPB O'Connor, Imaging Science and Biomedical Engineering, Stopford Building, University of Manchester, Oxford Road, Manchester, MI3 9PT, UK;

E-mail: james.o'connor@manchester.ac.uk

Received 16 August 2006; revised 7 November 2006; accepted 9 November 2006; published online 9 January 2007
}

promising, but its practical application is far from straightforward. The various analysis methods employed have considerable influence on the interpretation of derived parameters and their value as potential biomarkers and/or surrogate end points (Parker and Buckley, 2005). In this review, we outline the requirements of imaging biomarkers for clinical trials of novel agents and highlight relevant features of $\mathrm{T}_{1}$-weighted DCE-MRI that should be considered when implementing the technique in human studies. We then assess the clinical findings to date and outline future directions for DCE-MRI in anticancer drug development.

\section{GENERAL PRINCIPLES OF $\mathrm{T}_{1}$-WEIGHTED DCE-MRI IN CLINICAL TRIALS}

Dynamic contrast-enhanced magnetic resonance imaging is a noninvasive quantitative method of investigating microvascular structure and function by tracking the pharmacokinetics of injected low-molecular weight contrast agents as they pass through the tumour vasculature. The technique is sensitive to alterations in vascular permeability, extracellular extravascular and vascular volumes, and in blood flow (F). It does not involve ionising radiation, provides good spatial resolution and can be performed on standard specification 1.5 Tesla clinical systems. In this respect, MRI has practical advantages over computed tomography (CT) and positron emission tomography (PET) in evaluating angiogenesis.

In $\mathrm{T}_{1}$-weighted DCE-MRI, an intravenous bolus of gadolinium contrast agent enters tumour arterioles, passes through capillary beds and then drains via tumour veins. Gadolinium ions are paramagnetic and interact with nearby hydrogen nuclei to shorten $\mathrm{T}_{1}$-relaxation times in local tissue water. This causes increase in signal intensity on $\mathrm{T}_{1}$-weighted images to a variable extent within each voxel. The degree of signal enhancement is dependent on physiological and physical factors, including tissue perfusion, arterial input function (AIF) (AIF: the concentration-time course of contrast agent in the artery supplying the vascular bed), 
capillary surface area, capillary permeability and the volume of the extracellular extravascular leakage space (EES). $\mathrm{T}_{1}$-weighted DCE-MRI analysis generates parameters that represent one of, or combinations of these processes, and can be used to measure abnormalities in tumour vessel flow, blood volume, permeability, tortuosity and interstitial pressure (Figure 1). However signal enhancement will also be affected by contrast agent dose, the native $\mathrm{T}_{1}$-relaxation time of each tissue and choice of imaging sequences.

Dynamic contrast-enhanced magnetic resonance imaging strategies vary, but, in general, three types of imaging data are acquired. Initial images localise the tumour and provide anatomical information. Next, sequences that allow calculation of baseline tissue $T_{1}$-values before contrast agent administration are acquired to enable subsequent analysis. Finally, dynamic data are acquired every few seconds in $\mathrm{T}_{1}$-weighted images over a period of around 5-10 min. Dynamic sequences are subject to innate trade-offs between spatial resolution, temporal resolution (how quickly each image is acquired) and anatomical coverage. Fast $\mathrm{T}_{1}$-weighted spoiled gradient echo sequences are generally used as they allow good contrast medium sensitivity, high signal-to-noise ratio, adequate anatomical coverage and rapid data acquisition (Parker and Buckley, 2005)

\section{IMPORTANT CONSIDERATIONS FOR IMAGE ACQUISTION AND ANALYSIS}

Comprehensive discussion of the technical aspects of DCE-MRI image acquisition and analysis is beyond the scope of this paper However, some factors are briefly considered as selection of MRI sequences and data analysis methods determine not only the range of parameters available, but also their precise meaning.

\section{Analysis: descriptive or physiological?}

Several analysis methods can be applied to DCE-MRI data. Features of the signal intensity-time curve (e.g. gradient, overall shape, time to $90 \%$ maximum enhancement) represent

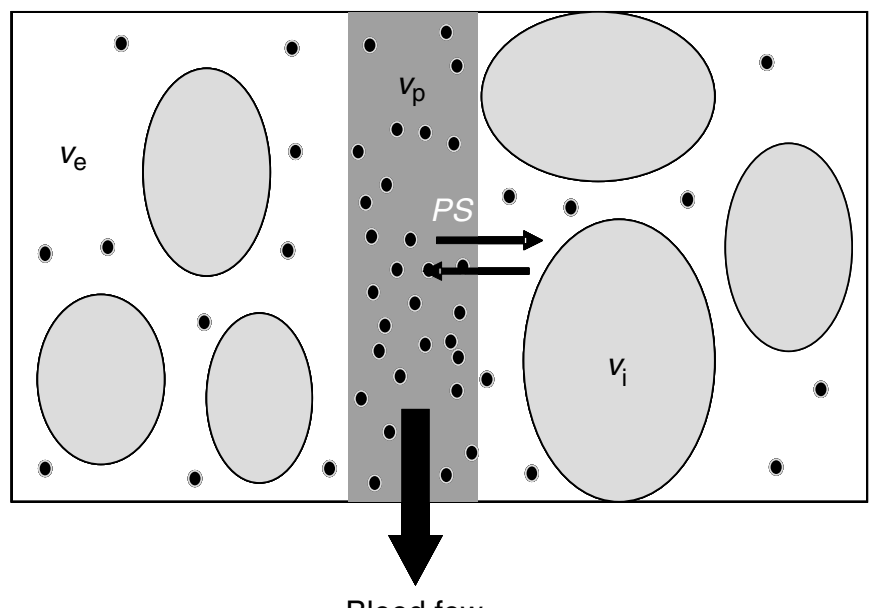

Blood fow

Figure I Compartmental modelling of the tumour microvasculature blood flows through the tumour enabling contrast media molecules (represented as black dots) to distribute in two potential compartments the blood plasma volume $v_{p}$ and the volume of the extravascular extracellular space $v_{e}$. Clinically available MRI contrast agents do not leak into the intracellular space $v_{i}$. Contrast agent leakage is governed by the concentration difference between the plasma and the extracellular extravascular space and by the permeability and surface area of the capillary endothelia, expressed as PS. simple descriptions of contrast agent distribution. However, these measures show considerable variation between acquisition method and individual examinations, making direct comparison between patients and trials difficult. Conversion of signal intensity into contrast agent concentration data allows more robust analysis of contrast agent kinetics. However, unlike dynamic CT or PET, the relationship between signal intensity and contrast agent concentration is not linear, making conversion of the signal intensity data far from straightforward (Tofts et al, 1999; Parker and Buckley, 2005).

Parameters that describe the shape of the contrast agent concentration-time curve represent a combination of flow, blood volume, vessel permeability and EES volume. One such quantity, the initial area under the contrast agent concentration-time curve (IAUC) is easy to calculate (model-free), reasonably reproducible and is routinely used as a biomarker in drugs trials. However, IAUC has a complicated and incompletely defined relationship with underlying tumour physiology and represents a composite of physiological processes (Tofts et al, 1999).

\section{Which model should be used?}

Pharmacokinetic models can be applied to contrast agent concentration data to enable estimates of physiological characteristics such as flow and capillary endothelial permeability. Modelled parameters are in theory more 'physiologically meaningful' than simple descriptors, such as IAUC, and are independent of acquisition protocol and solely reflect tissue characteristics. Thus, they are suitable measurements for multicentre studies with variation in image acquisition protocols and equipments (Leach et al, 2005).

Consensus opinion recommends that simple models describing the volume transfer coefficient of contrast between the blood plasma and the EES $\left(K^{\text {trans }}\right)$ and the size of the EES $\left(v_{\mathrm{e}}\right)$ should be used along with IAUC in assessing antiangiogenic and vascular disrupting agents in clinical trials (Leach et al, 2005). Other related measures such as the rate constant $\left(k_{\mathrm{ep}}\right)$, which describes the ratio of $K^{\text {trans }} / v_{\mathrm{e}}$ have also been used. Several models have been applied to clinical trial data to enable calculation of $K^{\text {trans }}$ and $v_{\mathrm{e}}$, many of which are equivalent (Larsson et al, 1990; Tofts and Kermode, 1991).

Changes in $F$, endothelial permeability and endothelial surface area produce changes in measurements of $K^{\text {trans }}$ (or an equivalent parameter, such as $K_{\mathrm{i}}$ ) in these models, and the specific contribution of the individual components cannot be identified. Importantly, the interpretation of $K^{\text {trans }}$ varies depending on the relationship between $F$ and capillary permeability-surface area product $(P S)$. When tissue contrast delivery is ample $(F \gg P S) K^{\text {trans }}$ represents the $P S$ per unit volume of tissue, for trans-endothelial transport between plasma and EES $\left(K^{\text {trans }} \sim P S\right)$. In limited perfusion $(P S \gg F) K^{\text {trans }}$ represents the $F$ per unit volume of tissue $\left(K^{\text {trans }} \sim F\right)$ (Tofts et al, 1999). In these simple models, both $K^{\text {trans }}$ and $v_{\mathrm{e}}$ calculation are relatively stable but lack physiological specificity.

Extensions of this model (Tofts 1997) are more complex, but enable calculation of blood plasma volume $\left(v_{\mathrm{p}}\right)$ and provide more accurate estimations of $K^{\text {trans }}$ and $v_{\mathrm{e}}$. More comprehensive models allow direct quantification of flow $(F)$, extraction fraction $(E), v_{\mathrm{e}}$ and mean capillary transit time $(\tau)$ (St Lawrence and Lee, 1998). Here, rather than defining the composite parameter $K^{\text {trans }}$, it is possible to separate $F$ and $P S$. However successful application of this model requires a temporal resolution in the order of $1 \mathrm{~s}$ to measure $\tau$ accurately, which limits its application in clinical trials (Jayson et al, 2005; Parker and Buckley, 2005).

$K^{\text {trans }}$ does not purely measure capillary permeability in any of these models (although it is often assumed to do so). Instead, its exact meaning depends on the kinetic model used for analysis. Changes in $K^{\text {trans }}$ may also represent different physiological 
processes in different individuals within the same patient cohort (e.g. reduction in $K^{\text {trans }}$ could represent reduced flow, reduced permeability or a combination of the two). The choice of analysis techniques is therefore not straightforward and reflects a compromise between parameters that are either relatively simple but poorly specific or physiologically congruent but less stable.

\section{Imaging protocol}

Incorporating DCE-MRI into clinical trials has required considerable technical expertise from basic scientists and clinicians in preparing nonstandard MRI sequences and in-house software for data analysis. Hence, early DCE-MRI studies varied in data acquisition and analysis methods, making comparison difficult and confusing. Multicentre trials require uniform image acquisition and analysis favouring reproducible machine-independent protocols. Such studies require careful quality control and are increasingly managed by contract research organisations specialising in advanced imaging applications. Multicentre DCE-MRI trial feasibility has been demonstrated by studies of the tyrosine kinase inhibitors AG-013736 (Liu et al, 2005) and BIBF 1120 (Padhani et al, 2006). At present, two baseline scans are recommended for all studies (to define parameter reproducibility) (Leach et al, 2005), but guidelines on the timing of MRI scanning and the choice of imaging parameters are less clear.

\section{Importance of the AIF}

In theory, the models described above require direct measurement of an AIF along with the tumour contrast agent concentrationtime course curve. These two functions are then used to quantify the passage of contrast agent through the tumour. Ideally, the AIF should be measured for each examination, as it varies between individuals and visits reflecting physiological variation in cardiac output, vascular tone, renal function and injection timing. Unfortunately, AIF measurement is technically demanding and, at best, produces an indirect measurement from a nearby large artery that may differ from the vessel supplying the tumour. Therefore, many groups use an idealised mathematical function instead, which allows far greater freedom in the imaging protocol by relaxing requirements on temporal resolution, slice positioning and sequence choice, but makes no attempt to reflect the true blood supply to the tumour at each examination (Parker and Buckley, 2005). Whichever technique is used, AIF measurement has a major impact on data analysis and clinical results - inaccuracy in the form or scale of the AIF affects the magnitude of all of the modelled parameters and their reproducibility.

\section{Region of interest and statistical analysis}

Data analysis is performed on a defined region of interest (ROI) that encompasses all or part of the tumour. A single averageenhancement curve can be extracted and used to generate values of parameters of interest (such as IAUC or $K^{\text {trans }}$ ), and the same parameters can then be compared following therapy (Dowlati et al, 2002; Morgan et al, 2003; Liu et al, 2005; Mross et al, 2005a; Thomas et al, 2005). This method ignores heterogeneity within the tumour. Alternatively, data can be extracted from each voxel within the ROI and summary statistics such as the mean and s.d., or median and interquartile range may be calculated (Jayson $e t a l$, 2002, 2005; Galbraith et al, 2003; Evelhoch et al, 2004). This second method can describe both normal and nonnormal data distributions and provides limited information regarding microvascular heterogeneity. In practice, both methods have been used in trials of antiangiogenic and vascular disrupting agents.

\section{Data quality}

Established and agreed policies for quality control are essential. $\mathrm{T}_{1}$-values should be checked against a reference range for the relevant magnetic field strength. Significant motion artefact, AIF and ROI definition and signal-to-noise ratio should be assessed and corrected. If correction is not possible, then corrupted datasets should be removed from subsequent analysis (Buonaccorsi et al, 2006). These considerations are important because they influence the accuracy of data (hence ensure quality control) and the significance/power of results (hence avoid dataset exclusion). In practice data are frequently suboptimal and can lead to trial data being excluded or of limited value (Eder et al, 2002; Morgan et al, 2003; Stevenson et al, 2003; Liu et al, 2005; O'Donnell et al, 2005).

\section{CLINICAL TRIALS TO DATE: METHODS AND RESULTS}

At the time of writing, 21 trials of antiangiogenic compounds (Eder et al, 2002; Jayson et al, 2002, 2005; Morgan et al, 2003; Thomas et al, 2003, 2005; Conrad et al, 2004; Medved et al, 2004; Overmoyer et al, 2004; Xiong et al, 2004; Drevs et al, 2005; Liu et al, 2005; Mross et al, 2005a, b; O'Donnell et al, 2005; O'Dwyer et al, 2005; Rosen et al, 2005, 2006; Padhani et al, 2006; Watson et al, 2006; Wedam et al, 2006) and six trials of vascular disrupting compounds (Dowlati et al, 2002; Galbraith et al, 2002, 2003; Stevenson et al, 2003; Evelhoch et al, 2004; McKeage et al, 2006) have employed $\mathrm{T}_{1}$-weighted DCE-MRI analysis and described their protocols and findings in enough detail to allow critical appraisal (Tables 1 and 2). Most were small cohort single-centre phase I trials in patients with advanced solid tumours, although a small number of phase II trials have incorporated DCE-MRI (Wedam et al, 2006). Marked variation in tumour size (Evelhoch et al, 2004), anatomy and pathophysiology and previous treatment (Morgan et al, 2003) have made data evaluation difficult and may have masked subtle drug effects, prompting a move towards stricter inclusion criteria (Morgan et al, 2003; Mross et al, 2005a; O'Dwyer et al, 2005) or using an intra-patient dose escalation design (Jayson et al, 2002; Stevenson et al, 2003).

\section{Biomarker evidence of drug effect - what does it mean?}

Dynamic contrast-enhanced magnetic resonance imaging parameters are subject to random error, biological variation and systemic inaccuracies that cause day-to-day variation in measured values. Many investigators consider a change in $K^{\text {trans }}$ of $\geqslant 40 \%$ as likely to represent a true difference in the parameter as some evidence suggests it correlates with disease stability/response, but in practice the confidence interval for each parameter depends on the choice of model, AIF methods and ROI definition (Roberts et al, 2006). Knowing the intrapatient variability for the study population is essential to have confidence that a parameter change is due to drug effect. Therefore, many (though not all) centres perform two baseline scans to measure reproducibility for each trial dataset, in accordance with published guidelines (Galbraith et al, 2003; Jayson et al, 2005; Padhani et al, 2006).

Evidence of drug efficacy has been demonstrated with DCE-MR1 in several trials of antiangiogenic drugs. Significant reductions in $K^{\text {trans }}$ have been reported in patients with advanced breast cancer receiving bevacizumab alone, implying reduction in $F$ and/or permeability. $K^{\text {trans }}$ reduction was increased following a further six cycles of bevacizumab with conventional chemotherapy. However changes in $K^{\text {trans }}$ did not predict response rate (RR) (Wedam et al, 2006). Several tyrosine kinase inhibitors, notably AG-013736 (Liu et al, 2005), BIBF1120 (Mross et al, 2005b) and AZD2171 (Drevs et al, 2005) have all shown dose-dependent reductions in $K^{\text {trans }}$ and IAUC without demonstrating clinical response. 
Table I Antiangiogenic agents evaluated by DCE-MRI in clinical trials

\begin{tabular}{|c|c|c|c|c|c|c|c|}
\hline Study & Agent & $\mathbf{N}$ & Tumour group $^{\mathbf{a}}$ & DCE-MRI biomarker & Evidence of drug effect & Inform dose & Predict outcome \\
\hline \multicolumn{8}{|c|}{ Anti-VEGF antibody } \\
\hline Jayson & HuMV833 & 20 & Mixed & $K^{\text {trans }}, k_{e p}, r B V$ & Reduction in $K_{\mathrm{ep}}$ but no dose relationship & No & No \\
\hline Overmoyer & Bevacizumab & 26 & Breast & $k_{\text {ep }}$ & Reduction in $\mathrm{K}_{\mathrm{ep}}$ & - & - \\
\hline Wedam & Bevacizumab & 20 & Breast & $K^{\text {trans }}, v_{\mathrm{e}}$ & $\downarrow K^{\text {trans }} 34 \% \mathrm{ECl}$ (BV alone) \& 75\% EC7 (BV+ cytotoxic) & No dose relationship & $K^{\text {trans }}$ did not predict RR \\
\hline \multicolumn{8}{|c|}{ Tyrosine kinase inhibitor } \\
\hline Conrad & PTK/ZK & 14 & GBM & $K_{i}, r B V$ & Dose-dependent $\downarrow K_{\mathrm{i}} \sim 40 \%$ at $\mathrm{d} 28$ & - & $K_{i}$ predicts progression \\
\hline Morgan & PTK/ZK & 26 & CRC & $K_{i}$ & Dose-dependent $\downarrow K_{\mathrm{i}}-58 \%$ in $\geqslant 1000 \mathrm{mg}$ at $\mathrm{d} 2,53 \%$ at $\mathrm{d} 28$ & Optimum $\geqslant 1000 \mathrm{mg}$ & $K_{i}$ predicts RR \& progression \\
\hline Mross & PTK/ZK & 27 & CRC/Breast & $K_{i}$ & Dose-dependent $\downarrow K_{\mathrm{i}}-54 \%$ in $\geqslant 1000 \mathrm{mg}$ at $\mathrm{d} 2,5 \mathrm{l} \%$ at $\mathrm{d} 28$ & Optimum $\geqslant 1000 \mathrm{mg}$ & $K_{i}$ predicts $R R \&$ progression \\
\hline Thomas & PTK/ZK & 35 & Mixed & $K_{i}$ & Dose-dependent $\downarrow K_{i}-46 \%$ in $\geqslant 1000 \mathrm{mg}$ at d2, 40\% at d28 & Optimum $\geqslant 1000 \mathrm{mg}$ & $K_{i}$ predicts RR \& progression \\
\hline Drevs & AZD217I & 24 & Mixed & IAUC & $\downarrow$ IAUC of $\geqslant 40 \%$ in five out of seven patients & Effective $20-45 \mathrm{mg}$ & - \\
\hline Medved & SU 5146 & 19 & Mixed & IAUC & Progressive disease despite $\downarrow$ IAUC & - & - \\
\hline O'Donnell & SU 5146 & 24 & Mixed & $K^{\text {trans }}, v_{\mathrm{e}}$ & No consistent relationship with clinical measures & No dose relationship & - \\
\hline Liu & AG-013736 & 17 & Mixed & IAUC, $K^{\text {trans }}$ & Dose-dependent $\downarrow$ IAUC, $K^{\text {trans }} \mathrm{d} 2$ and $\mathrm{d} 28$. Effect lost d56 & - & - \\
\hline O'Dwyer & BAY 43-9006 & 12 & Renal CC & $K^{\text {trans }}, v_{e}$ & Mean reduction $K^{\text {trans }} 61 \%$ & & $K^{\text {trans }}$ predicts RR \& PFS \\
\hline Rosen & AMG-706 & 18 & Mixed & IAUC & $\downarrow \mid A \cup C$ of $\leqslant 61 \%$ & - & - \\
\hline Rosen & BMS-582664 & 7 & Mixed & $K^{\text {trans }}$ & $\downarrow K^{\text {trans }} \geqslant 40 \%$ in $600-800 \mathrm{mg}$ cohort & Effective $600-800 \mathrm{mg}$ & - \\
\hline Xiong & SU6668 & 4 & Mixed & IAUC, slope & None & No & No \\
\hline Mross & BIBFIII20 & 27 & Mixed & IAUC, $K_{\mathrm{i}}$ & Reduced IAUC \& $K_{i} \geqslant 40 \%$ in higher dose groups & No & - \\
\hline Padhani & BIBFII 20 & 35 & Mixed & IAUC, $K^{\text {trans }}, K_{\text {ep }}$ & No consistent relationship with clinical measures & No & - \\
\hline \multicolumn{8}{|c|}{ Anti-PDGFR- $\beta$ antibody Fab' } \\
\hline Jayson & CDP860 & 8 & CRClOvary & IAUC, $K^{\text {trans }}, v_{e}, v_{p}$ & $\uparrow$ vascularised tumour volume in some patients & No & No \\
\hline \multicolumn{8}{|c|}{ Recombinant human endostatin } \\
\hline Eder & rhEndostatin & 10 & Mixed & $K^{\text {trans }}, v_{e}$ & None & No & No correlation with PFS or OS \\
\hline Thomas & rhEndostatin & 21 & Mixed & No detail provided & None & No & No correlation with TTP \\
\hline \multicolumn{8}{|l|}{ Anti- $\alpha v$ integrin } \\
\hline Watson & CNTO95 & 22 & Mixed & IAUC, $K^{\text {trans }}, v_{e}, v_{p}$ & No consistent relationship with clinical measures & No & - \\
\hline
\end{tabular}

$\mathrm{CRC}=$ colorectal; $\mathrm{d}=$ day; $\mathrm{DCE}-\mathrm{MRI}=$ Dynamic contrast-enhanced magnetic resonance imaging; $\mathrm{EC}=$ end cycle; $\mathrm{GBM}=$ glioblastoma multiforme; IAUC = initial area under the contrast agent concentration-time curve; $K_{i}=$ uni-

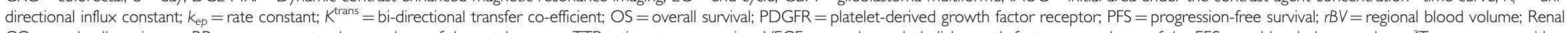
$\mathrm{CC}=$ renal cell carcinoma; $\mathrm{RR}=$ response rate; slope $=$ slope of the uptake curve; $T \mathrm{TP}=$ time to progression; $\mathrm{VEGF}=$ vascular endothelial growth factor; $v_{\mathrm{e}}=$ volume of the EES; $v_{p}=$ blood plasma volume ${ }^{\mathrm{a}} \mathrm{Tumours}$ were either mixed solid group, or breast, glioblastoma multiforme (GBM), colorectal (CRC), renal cell carcinoma (Renal CC) or epithelial ovarian (Ovary). 
Few trials have demonstrated a relationship between DCE-MRI biomarker and clinical outcome measure. Correlation of $K^{\text {trans }}$ reduction and RR and progression-free survival (PFS) has been shown with BAY 43-9006 (O'Dwyer et al, 2005). Reduction, in $K_{\mathrm{i}}$ of $\geqslant 40 \%$ has predicted which patients progressed with glioblastoma multiforme in a trial of PTK787/ZK222584 (PTK/ZK) (Conrad et al, 2004). Changes in $K^{\text {trans }}$ following trials of AZD2171 (Drevs et al, 2005) and BMS-582664 (Rosen et al, 2006) have helped define the effective dose to take into phase II studies.

The studies outlined above show that DCE-MRI biomarkers can provide early indicators of efficacy, dose and outcome. However, such findings do not guarantee success in phase III development. Three related trials of PTK/ZK reported promising early results with DCE-MRI biomarkers. Patients with colorectal carcinoma liver metastases (mixture of disease stage and extent of prior therapy) showed a dose-dependent reduction in $K_{\mathrm{i}}$ of $43 \%$ at day 2, which was greatest in higher-dose groups ( $\geqslant 1000 \mathrm{mg}$ once daily), where $K_{\mathrm{i}}$ was reduced by $58 \%$ on day 2 and $53 \%$ at the end of cycle 1 (EC1). Reduction in tumour enhancement at day 2 and EC1 (measured by reduction in $K_{\mathrm{i}}$ from baseline) predicted disease progression and was positively correlated with reduction in tumour size (Morgan et al, 2003). Statistically significant dosedependent changes in $K_{\mathrm{i}}$ from baseline at day 2 and day 28 were identified in two subsequent studies of PTK/ZK in patients with mixed solid tumours ( $\geqslant 1000 \mathrm{mg}$ once daily) (Mross et al, 2005a; Thomas et al, 2005). A biologically active dose of $1000 \mathrm{mg}$ was identified in all three studies.

Two subsequent randomised phase III trials of PTK/ZK with standard treatment in patients with metastatic colorectal carcinoma suggest that DCE-MRI parameter changes may be necessary but not sufficient biomarkers of drug efficacy. Oral PTK/ZK (1250 mg q.d.s) or placebo were administered with oxaloplatin/ 5-flurouracil/leucovorin (FOLFOX4) as first-line (CONFIRM-1) (Hecht et al, 2005) or second-line therapy (CONFIRM-2) (Koehne et al, 2006). Analysis of PFS in CONFIRM-1 did not achieve statistical significance. Overall survival (OS) data are awaited. Response rate between trial arms were not significantly different and the primary endpoint of OS was not met in CONFIRM-2, although PFS was significantly longer in the PTK/ZK arm (5.5 vs 4.1 months; hazard ratio $0.83 ; P=0.026$ ).

Fewer trials of vascular disrupting agents have incorporated DCE-MRI biomarkers. Evidence of drug efficacy has been shown in the flavonoid DMXAA (Galbraith et al, 2002), the antitubulin agent CA-4-P (Galbraith et al, 2003) and the colchicine analogue ZD6126 (Evelhoch et al, 2004). Evaluation of CA-4-P helped define the biologically active and maximum tolerated doses.

\section{Negative results - how confident are we?}

Three factors are imperative if DCE-MRI biomarkers are to detect true negative results and hence increase the value of DCE-MRI in evaluating antiangiogenic and vascular disrupting agents. Firstly, correct scan schedule is required. For example, vascular disrupting agent drug effects are typically seen within hours of administration and may be lost within $24 \mathrm{~h}$. Thus, DCE-MRI trials of vascular disrupting agents require imaging at baseline, $4-6$ and at $24 \mathrm{~h}$. In contrast, antiangiogenic drug effects typically occur within days to weeks and may persist for weeks to months, so that imaging must be performed at these time points.

Secondly, correct and robust parameters must be selected for each compound and used appropriately. Most studies employ changes in mean or median IAUC and $K^{\text {trans }}$ irrespective of the proposed drug mechanism. Although changes in $K^{\text {trans }}$ may be meaningful in trials of VEGF/VEGF-receptor inhibitors, its usefulness in evaluating other compounds is less clear. Parameters, such as change in relative enhancing fraction within the total tumour volume, influenced by altered interstitial pressure, may be more informative in assessment of platelet-derived growth 
factor/platelet-derived growth factor receptor inhibitors (Jayson et al, 2005). Parameter changes require correct interpretation, as for example $K^{\text {trans }}$ changes may reflect altered flow or vascular permeability. Data quality (minimal motion, well-defined AIF and reproducible analysis) must be sufficient to allow measured parameters changes to be detected.

Finally, data analysis is crucial and must be critically appraised in each trial. Current methods that produce summary parameters oversimplify data and may mask critical information concerning tumour heterogeneity. Alternative methods of data evaluation, such as histogram analysis (Watson et al, 2006) may have a role in demonstrating changes in heterogeneity. Equally, tumour subregions may require separate analysis in order to detect subtle drug effects (e.g. rim-core differential of vascular disrupting agent action) (Walker-Samuel et al, 2006), which may otherwise be obscured and be reported as false negative results.

\section{CONCLUSIONS AND FUTURE CHALLENGES}

Magnetic resonance imaging biomarkers of tumour angiogenesis require validation, ideally against clinical outcome measures such as OS, PFS or RR. At present, an insufficient number of clinical studies have correlated changes in $K^{\text {trans }}$, IAUC or other DCE-MRI biomarkers with clinical outcome to allow their adoption as established surrogate end points of drug effects. Validation against

\section{REFERENCES}

Baluk P, Hashizume H, McDonald DM (2005) Cellular abnormalities of blood vessels as targets in cancer. Curr Opin Genet Dev 15: $102-111$

Buonaccorsi GA, Roberts C, Cheung S, Watson Y, O'Connor JP, Davies K, Jackson A, Jayson GC, Parker GJ (2006) Comparison of the performance of tracer kinetic model-driven registration for dynamic contrast enhanced MRI using different models of contrast enhancement. Acad Radiol 13: $1112-1123$

Conrad C, Friedman H, Reardon D, Provenzale J, Jackson E, Serajuddin H, Laurent D, Chen B, Yung WKA (2004) A phase I/II trial of single-agent PTK 787/ZK 222584 (PTK/ZK), a novel, oral angiogenesis inhibitor, in patients with recurrent glioblastoma multiforme (GBM). J Clin Oncol (Meetings Abstracts) 22(14S): 1512

Dowlati A, Robertson K, Cooney M, Petros WP, Stratford M, Jesberger J Rafie N, Overmoyer B, Makkar V, Stambler B, Taylor A, Waas J, Lewin JS, McCrae KR, Remick SC (2002) A phase I pharmacokinetic and translational study of the novel vascular targeting agent combretastatin a-4 phosphate on a single-dose intravenous schedule in patients with advanced cancer. Cancer Res 62: 3408-3416

Drevs J, Medinger M, Mross K, Zirrgiebel U, Strecker R, Unger C, Puchalski TA, Fernandes N, Roberston J, Siegert P (2005) Phase I clinical evaluation of AZD2171, a highly potent VEGF receptor tyrosine kinase inhibitor, in patients with advanced tumors. J Clin Oncol (Meetings Abstracts) 23(16S): 3002

Eder Jr JP, Supko JG, Clark JW, Puchalski TA, Garcia-Carbonero R, Ryan DP, Shulman LN, Proper J, Kirvan M, Rattner B, Connors S, Keogan MT Janicek MJ, Fogler WE, Schnipper L, Kinchla N, Sidor C, Phillips E, Folkman J, Kufe DW (2002) Phase I clinical trial of recombinant human endostatin administered as a short intravenous infusion repeated daily. J Clin Oncol 20: $3772-3784$

Evelhoch JL, LoRusso PM, He Z, DelProposto Z, Polin L, Corbett TH, Langmuir P, Wheeler C, Stone A, Leadbetter J, Ryan AJ, Blakey DC, Waterton JC (2004) Magnetic resonance imaging measurements of the response of murine and human tumors to the vascular-targeting agent ZD6126. Clin Cancer Res 10: 3650 - 3657

Galbraith SM, Maxwell RJ, Lodge MA, Tozer GM, Wilson J, Taylor NJ, Stirling JJ, Sena L, Padhani AR, Rustin GJ (2003) Combretastatin A4 phosphate has tumor antivascular activity in rat and man as demonstrated by dynamic magnetic resonance imaging. J Clin Oncol 21: $2831-$ 2842

Galbraith SM, Rustin GJ, Lodge MA, Taylor NJ, Stirling JJ, Jameson M, Thompson P, Hough D, Gumbrell L, Padhani AR (2002) Effects of 5,6- histopathology biomarkers such as microvascular density is problematic in DCE-MRI, where micrometer scale biopsy changes must be compared against voxel resolution in millimetres. Nonetheless, histopathology validation is important and can substantiate the use of a biomarker in phase I/II trials. Both animal models and clinical studies are likely to be required to achieve comprehensive validation (Jayson and Waterton, 2005; Leach et al, 2005).

Dynamic contrast-enhanced magnetic resonance imaging is considerably more complex than conventional anatomical imaging. Acquisition and analysis protocols place a significant demand on both patients and staff. However, initial studies show that the technique is feasible, and $\mathrm{T}_{1}$-weighted DCE-MRI parameters have provided considerable noninvasive information concerning tumour biology and response to treatment. Future developments in image acquisition and analysis are evolving rapidly and may further increase the potential that DCE-MRI has to play in future assessment, diagnosis and follow-up of cancer.

\section{ACKNOWLEDGEMENTS}

JOC is funded by a Cancer Research UK Clinical Research Training Fellowship Grant, ref C19221/A6086. Cancer Research UK is a charity registered in the United Kingdom, No. 1089464. dimethylxanthenone-4-acetic acid on human tumor microcirculation assessed by dynamic contrast-enhanced magnetic resonance imaging. J Clin Oncol 20: 3826-3840

Hecht J, Trarbach T, Jaeger E, Hainsworth J, Wolff R, Lloyd K, Bodoky G, Borner M, Laurent D, Jacques C (2005) A randomized, double-blind, placebo-controlled, phase III study in patients (Pts) with metastatic adenocarcinoma of the colon or rectum receiving first-line chemotherapy with oxaliplatin/5-fluorouracil/leucovorin and PTK787/ ZK 222584 or placebo (CONFIRM-1). J Clin Oncol (Meetings Abstracts) 23(16S): 3

Hurwitz H, Fehrenbacher L, Novotny W, Cartwright T, Hainsworth J, Heim W, Berlin J, Baron A, Griffing S, Holmgren E, Ferrara N, Fyfe G, Rogers B, Ross R, Kabbinavar F (2004) Bevacizumab plus irinotecan, fluorouracil, and leucovorin for metastatic colorectal cancer. $N$ Engl J Med 350: 2335-2342

Jayson GC, Parker GJ, Mullamitha S, Valle JW, Saunders M, Broughton L, Lawrance J, Carrington B, Roberts C, Issa B, Buckley DL, Cheung S, Davies K, Watson Y, Zinkewich-Peotti K, Rolfe L, Jackson A (2005) Blockade of platelet-derived growth factor receptor-beta by CDP860, a humanized, PEGylated di-Fab', leads to fluid accumulation and is associated with increased tumor vascularized volume. J Clin Oncol 23: 973-981

Jayson GC, Waterton JC (2005) Applications of dynamic contrast-enhanced MRI in oncology drug development. In Dynamic Contrast-enhanced Magnetic Resonance Imaging in Oncology Jackson A, Buckley DL, Parker GJM (eds). pp 281-297. Berlin: Springer

Jayson GC, Zweit J, Jackson A, Mulatero C, Julyan P, Ranson M, Broughton L, Wagstaff J, Hakannson L, Groenewegen G, Bailey J, Smith N, Hastings D, Lawrance J, Haroon H, Ward T, McGown AT, Tang M, Levitt D, Marreaud S, Lehmann FF, Herold M, Zwierzina H (2002) Molecular imaging and biological evaluation of HuMV833 anti-VEGF antibody: implications for trial design of antiangiogenic antibodies. J Natl Cancer Inst 94: $1484-1493$

Koehne C, Bajetta E, Lin E, Van Cutsem E, Hecht J, Douillard J, Moore M, Germond C, Laurent D, Jacques C (2006) Results of an interim analysis of a multinational randomized, double-blind, phase III study in patients (pts) with previously treated metastatic colorectal cancer (mCRC) receiving FOLFOX4 and PTK787/ZK 222584 (PTK/ZK) or placebo (CONFIRM 2). J Clin Oncol (Meetings Abstracts) 24(18S): 3508

Larsson HB, Stubgaard M, Frederiksen JL, Jensen M, Henriksen O, Paulson OB (1990) Quantitation of blood-brain barrier defect by 
magnetic resonance imaging and gadolinium-DTPA in patients with multiple sclerosis and brain tumors. Magn Reson Med 16: 117-131

Leach MO, Brindle KM, Evelhoch JL, Griffiths JR, Horsman MR, Jackson A, Jayson GC, Judson IR, Knopp MV, Maxwell RJ, McIntyre D, Padhani AR, Price P, Rathbone R, Rustin GJ, Tofts PS, Tozer GM, Vennart W, Waterton JC, Williams SR, Workman P (2005) The assessment of antiangiogenic and antivascular therapies in early-stage clinical trials using magnetic resonance imaging: issues and recommendations. $\mathrm{Br} \mathrm{J}$ Cancer 92: $1599-1610$

Liu G, Rugo HS, Wilding G, McShane TM, Evelhoch JL, Ng C, Jackson E, Kelcz F, Yeh BM, Lee Jr FT, Charnsangavej C, Park JW, Ashton EA, Steinfeldt HM, Pithavala YK, Reich SD, Herbst RS (2005) Dynamic Contrast-enhanced Magnetic Resonance Imaging as a pharmacodynamic measure of response after acute dosing of AG-013736, an oral angiogenesis inhibitor, in patients with advanced solid tumors: results from a phase I study. J Clin Oncol 23: 5464-5473

McKeage MJ, Fong P, Jeffery M, Baguley BC, Kestell P, Ravic M, Jameson MB (2006) 5,6-Dimethylxanthenone-4-acetic acid in the treatment of refractory tumors: a phase I safety study of a vascular disrupting agent. Clin Cancer Res 12: 1776-1784

Medved M, Karczmar G, Yang C, Dignam J, Gajewski TF, Kindler H, Vokes E, MacEneany P, Mitchell MT, Stadler WM (2004) Semiquantitative analysis of dynamic contrast enhanced MRI in cancer patients: variability and changes in tumor tissue over time. J Magn Reson Imaging 20: $122-128$

Morgan B, Thomas AL, Drevs J, Hennig J, Buchert M, Jivan A, Horsfield MA, Mross K, Ball HA, Lee L, Mietlowski W, Fuxuis S, Unger C, O’Byrne K, Henry A, Cherryman GR, Laurent D, Dugan M, Marme D, Steward WP (2003) Dynamic contrast-enhanced magnetic resonance imaging as a biomarker for the pharmacological response of PTK787/ZK 222584, an inhibitor of the vascular endothelial growth factor receptor tyrosine kinases, in patients with advanced colorectal cancer and liver metastases: results from two phase I studies. J Clin Oncol 21: 3955-3964

Mross K, Drevs J, Muller M, Medinger M, Marme D, Hennig J, Morgan B, Lebwohl D, Masson E, Ho YY, Gunther C, Laurent D, Unger C (2005a) Phase I clinical and pharmacokinetic study of PTK/ZK, a multiple VEGF receptor inhibitor, in patients with liver metastases from solid tumours. Eur J Cancer 41: 1291 - 1299

Mross KB, Gmehling D, Frost A, Baas F, Strecker R, Hennig J, Stopfer P, Stefanic M, Stehle G, de Rossi L (2005b) A clinical phase I, pharmacokinetic (PK), and pharmacodynamic study of twice daily BIBF 1120 in advanced cancer patients. J Clin Oncol (Meetings Abstracts) 23(16S): 3031

O'Donnell A, Padhani A, Hayes C, Kakkar AJ, Leach M, Trigo JM, Scurr M, Raynaud F, Phillips S, Aherne W, Hardcastle A, Workman P, Hannah A, Judson I (2005) A phase I study of the angiogenesis inhibitor SU5416 (semaxanib) in solid tumours, incorporating dynamic contrast MR pharmacodynamic end points. Br J Cancer 93: 876-883

O’Dwyer PJ, Rosen M, Gallagher M, Schwartz B, Flaherty KT (2005) Pharmacodynamic study of BAY 43-9006 in patients with metastatic renal cell carcinoma. J Clin Oncol (Meetings Abstracts) 23(16S): 3005

Overmoyer B, Silverman P, Leeming R, Shenk R, Lyons J, Ziats N, Jesberger J, Dumadag L, Remick S, Chen H (2004) Phase II trial of neoadjuvant docetaxel with or without bevacizumab in patients with locally advanced breast cancer. J Clin Oncol (Meeting Abstracts) 22(14S): 727

Padhani AR, Taylor NJ, d'Arcy JA, Walker-Samuel S, Collins DJ, Knowles B, Stirling JJ, Wallace T, Koh D-M, Tang A, Lee C, Temple G, Rustin GJ, Leach MO (2006) Dynamic MRI evaluation of the triple receptor tyrosine kinase inhibitor BIBF 1120 in patients with advanced solid tumours. Proc Int Soc Mag Reson Med 14: 765

Parker GJM, Buckley DL (2005) Tracer kinetic modelling for T1-weighted DCE-MRI. In Dynamic contrast-enhanced Magnetic Resonance Imaging in Oncology Jackson A, Buckley DL, Parker GJM (eds), pp 81 - 92. Berlin: Springer

Roberts C, Issa B, Stone A, Jackson A, Waterton JC, Parker GJ (2006) Comparative study into the robustness of compartmental modeling and model-free analysis in DCE-MRI studies. J Magn Reson Imaging 23: 554 563

Rosen LS, Kurzrock R, Jackson E, Wathen L, Parson M, Eschenberg M, Mulay M, Purdom M, Yan L, Herbst RS (2005) Safety and pharmacokinetics of AMG 706 in patients with advanced solid tumors. J Clin Oncol (Meetings Abstracts) 23(16S): 3013

Rosen LS, Wilding G, Sweeney C, Casale D, Kollia G, Wu C, Ayers M, Hill C, Galbraith SM (2006) Phase I dose escalation study to determine the safety, pharmacokinetics and pharmacodynamics of BMS-582664, a VEGFR/FGFR inhibitor in patients with advanced/metastatic solid tumors. J Clin Oncol (Meetings Abstracts) 24(18S): 3051

St Lawrence KS, Lee TY (1998) An adiabatic approximation to the tissue homogeneity model for water exchange in the brain: I. Theoretical derivation. J Cereb Blood Flow Metab 18: 1365-1377

Stevenson JP, Rosen M, Sun W, Gallagher M, Haller DG, Vaughn D, Giantonio B, Zimmer R, Petros WP, Stratford M, Chaplin D, Young SL, Schnall M, O'Dwyer PI (2003) Phase I trial of the antivascular agent combretastatin A4 phosphate on a 5-day schedule to patients with cancer: magnetic resonance imaging evidence for altered tumor blood flow. J Clin Oncol 21: 4428-4438

Thomas AL, Morgan B, Horsfield MA, Higginson A, Kay A, Lee L, Masson E, Puccio-Pick M, Laurent D, Steward WP (2005) Phase I study of the safety, tolerability, pharmacokinetics, and pharmacodynamics of PTK787/ZK 222584 administered twice daily in patients with advanced cancer. J Clin Oncol 23: 4162-4171

Thomas JP, Arzoomanian RZ, Alberti D, Marnocha R, Lee F, Friedl A, Tutsch K, Dresen A, Geiger P, Pluda J, Fogler W, Schiller JH, Wilding G (2003) Phase I pharmacokinetic and pharmacodynamic study of recombinant human endostatin in patients with advanced solid tumors. J Clin Oncol 21: 223-231

Tofts PS (1997) Modeling tracer kinetics in dynamic Gd-DTPA MR imaging. J Magn Reson Imaging 7: $91-101$

Tofts PS, Brix G, Buckley DL, Evelhoch JL, Henderson E, Knopp MV, Larsson HB, Lee TY, Mayr NA, Parker GJ, Port RE, Taylor J, Weisskoff RM (1999) Estimating kinetic parameters from dynamic contrastenhanced $\mathrm{T}(1)$-weighted MRI of a diffusable tracer: standardized quantities and symbols. J Magn Reson Imaging 10: 223-232

Tofts PS, Kermode AG (1991) Measurement of the blood-brain barrier permeability and leakage space using dynamic MR imaging. 1. Fundamental concepts. Magn Reson Med 17: 357-367

Walker-Samuel S, Taylor NJ, Padhani AR, Leach MO, Collins DJ (2006) The effect of heterogeneous tumour enhancement on the assessment of response to treatment. Proc Int Soc Mag Reson Med 14: 761

Watson Y, Cheung S, Roberts C, Buonaccorsi G, Davies K, Jackson A, Ton C, Broughton L, Power F, Jayson G, Lang Z, Beckman R, Parker G (2006) Prognostic power of DCE-MRI heterogeneity analysis in patients with advanced solid tumors. Proc Int Soc Mag Reson Med 14: 755

Wedam SB, Low JA, Yang SX, Chow CK, Choyke P, Danforth D, Hewitt SM, Berman A, Steinberg SM, Liewehr DJ, Plehn J, Doshi A, Thomasson D, McCarthy N, Koeppen H, Sherman M, Zujewski J, Camphausen K, Chen H, Swain SM (2006) Antiangiogenic and antitumor effects of bevacizumab in patients with inflammatory and locally advanced breast cancer. J Clin Oncol 24: 769-777

Xiong HQ, Herbst R, Faria SC, Scholz C, Davis D, Jackson EF, Madden T, McConkey D, Hicks M, Hess K, Charnsangavej CA, Abbruzzese JL (2004) A phase I surrogate endpoint study of SU6668 in patients with solid tumors. Invest New Drugs 22: 459-466 